\title{
A NEW FORMULATION OF MULTI-ZONE COMBUSTION ENGINE MODELS
}

\author{
Ylva Nilsson and Lars Eriksson \\ Vehicular Systems \\ Dept. of Electrical Engineering, Linköping University \\ SE-581 83 Linköping, Sweden \\ Email: ylvaneisy.liu.se, larerdisy.liu.se
}

\begin{abstract}
Cylinder pressure simulation has grown to become an important tool when developing and evaluating new engine concepts and control strategies. A new formulation of zero-dimensional multi-zone models is developed and described. A general model structure is formulated that rely on a set of differential algebraic equations that are easy to solve. The selected formulation also results in models that are easy to scale, i.e. add new zones, and to increase complexity, which is a result of the selected structure. A number of important issues that can cause problems when simulating the model are treated. It is shown: a) How a new zone is initialized. b) How variables of varying magnitude can be scaled to avoid numerical difficulties. c) How numerical errors accumulated during the simulation can be reduced by using a set of consistency equations.
\end{abstract}

Keywords: multi-zone, simulation, engine, cylinder pressure

\section{INTRODUCTION}

Cylinder pressure simulation is an important tool when developing and evaluating new engine concepts and control strategies. Cylinder pressure models are derived from the first law of thermodynamics which connects the thermodynamic properties such as pressure and temperature to the engine revolutions. These models have been developed with different complexity and are used in a wide range of applications from analysis of engine data from test-benches to predicting the performance of new engine concepts. The models are currently too complex to be implemented directly in engine management systems and are mainly used in laboratories and for off-line simulations. However, the computational power of the computerized control systems is steadily increasing and will in the foreseeable future enable realtime implementations of these models. This opens up possibilities for direct monitoring and control of the combustion process.

Here, a new formulation of zero-dimensional multizone models is developed and described. The model is: a) zero dimensional since it does not take spatial variations in the zones into account, b) multi zone since the cylinder is divided into a number of zones. These are homogeneous, isolated, and have their individual thermodynamic properties. It should be mentioned that the underlying structure of the model is the same as most other models presented in the literature, so the model is not new in itself; instead, it is the formulation that is new. A general model structure is formulated that rely on a set of differential algebraic equations that are easy to solve. The selected formulation also results in models that are easy to scale, i.e. add new zones, and to increase complexity, which is a result of the selected structure.

A wide variety of models have been described in the literature (Ramos, 1989; Keating, 1993; Turns, 2000), and the user of the model can choose among a number of models of varying complexity and select the model best suited for the application. This possibility is further enhanced with the new formulation. In addition, this paper also shows how to handle a number of practical 
problems that arise when the general model is used in a simulation.

\section{THE MULTI-ZONE COMBUSTION MODEL}

Consider a system with $N$ zones. The whole system has a certain volume, pressure and mass $(V, p$, and $m)$. The pressure is assumed to be homogeneous throughout the combustion chamber. Each zone has its own volume, temperature, mass $\left(V_{i}, T_{i}\right.$, and $m_{i}$ for zone $\left.i\right)$ and gas composition. The change in system volume and the mass transfer from zone $i$ to zone $j$ ( $d V$ and $d m_{i j}$ respectively) are assumed to be known, and the mass of a zone can easily be determined by integrating the mass flows in and out of the zone. The change of the remaining quantities $\left(p, T_{i}\right.$ and $\left.V_{i}, i=1,2, \ldots, N\right)$ are unknown and are to be determined.

The system consists of $\mathrm{N}$ zones. Therefore we know that the sum of all $V_{i}, i=1,2, \ldots, N$, must be the same as the volume of the whole system, i.e.

$$
\sum_{i} d V_{i}=d V
$$

The energy balance equation for zone $i$ is given by (Sonntag et al., 1998):

$$
d U_{i}=-d Q_{i}-d W_{i}+\sum_{j \neq i} d m_{i j} h_{i j}
$$

where $d Q_{i}$ is the heat transfer from the zone, $d W_{i}$ the work done by the gas which is $d W_{i}=p d v_{i}$, and $h_{i j}$ the enthalpy of the gas that enters the zone - or leaves if $d m_{i j}$ is negative. The internal energy is:

$$
d U_{i}=m_{i} d u_{i}+\sum_{j \neq i} u_{i} d m_{i j}
$$

Since the gas composition is not necessarily fix, $d u_{i}$ depends on both temperature and pressure:

$$
d u_{i}\left(p, T_{i}\right)=\left(\frac{\partial u_{i}}{\partial p}\right)_{T_{i}} d p+\left(\frac{\partial u_{i}}{\partial T_{i}}\right)_{p} d T_{i}
$$

From the second law of thermodynamics:

$$
d U_{i}=T_{i} d S_{i}-p d V_{i}
$$

and the Maxwell relations (Finn, 1998), the first term in (4) can be derived:

$$
\begin{aligned}
\left(\frac{\partial u_{i}}{\partial p}\right)_{T_{i}} & =T_{i}\left(\frac{\partial s_{i}}{\partial p}\right)_{T_{i}}-p\left(\frac{\partial v_{i}}{\partial p}\right)_{T_{i}}= \\
= & -T_{i}\left(\frac{\partial v_{i}}{\partial T_{i}}\right)_{p}-p\left(\frac{\partial v_{i}}{\partial p}\right)_{T_{i}}= \\
& =-\frac{T_{i}^{2}}{p}\left(\frac{\partial R_{i}}{\partial T_{i}}\right)_{p}-T_{i}\left(\frac{\partial R_{i}}{\partial p}\right)_{T_{i}}
\end{aligned}
$$

The second term in (4) is

$$
\left(\frac{\partial u_{i}}{\partial T_{i}}\right)_{p}=\left(\frac{\partial h_{i}}{\partial T_{i}}\right)_{p}-R_{i}-T_{i}\left(\frac{\partial R_{i}}{\partial T_{i}}\right)_{p}
$$

and according to the definition $\left(\frac{\partial h_{i}}{\partial T_{i}}\right)_{p}=c_{p, i}$ (Kittel and Kroemer, 1980). Equation (2) together with (3), (4), (5), and (6) gives:

$$
p d V_{i}+c_{i} d p+d_{i} d T_{i}=-d Q_{i}+\sum_{j \neq i}\left(h_{i j}-h_{i}+R_{i} T_{i}\right) d m_{i j}
$$

where

$$
\begin{aligned}
& c_{i}=-m_{i} T_{i}\left(\frac{T_{i}}{p}\left(\frac{\partial R_{i}}{\partial T_{i}}\right)_{p}+\left(\frac{\partial R_{i}}{\partial p}\right)_{T_{i}}\right) \\
& d_{i}=m_{i}\left(c_{p}-R_{i}-T_{i}\left(\frac{\partial R_{i}}{\partial T_{i}}\right)_{p}\right)
\end{aligned}
$$

The ideal gas law in differentiated form gives the last equation needed to get an unambiguous equation system:

$$
p d V_{i}+a_{i} d p+b_{i} d T_{i}=R T \sum_{j \neq i} d m_{i j}
$$

where

$$
\begin{aligned}
a_{i} & =V_{i}\left(1-\frac{p}{R_{i}}\left(\frac{\partial R_{i}}{\partial p}\right)_{T_{i}}\right) \\
b_{i} & =-m_{i}\left(R_{i}+T_{i}\left(\frac{\partial R_{i}}{\partial T_{i}}\right)_{p}\right)
\end{aligned}
$$

Equation (1), (7), and (8) make a system of $2 N+1$ ordinary differential equations. In these equations, the right hand side of the equality signs are known from the previous step, while the differentials on the left hand side are to be determined. The differentials on the left hand side are linear in the unknowns, and the system can thus be expressed

$$
\mathbb{A} d x=\mathbb{B}
$$

where $d x$ is a vector containing all the unknowns. Selecting $d x$ to

$$
d x=\left[d p d V_{1} d T_{1} \ldots d V_{N} d T_{N}\right]^{T}
$$

yields the following $\mathbb{A}$ - and $\mathbb{B}$-matrix:

$$
\mathbb{A}=\left(\begin{array}{cccccc}
0 & 1 & 0 & \ldots & 1 & 0 \\
a_{1} & p & b_{1} & \ldots & 0 & 0 \\
c_{1} & p & d_{1} & \ldots & 0 & 0 \\
\vdots & \vdots & \vdots & \ddots & \vdots & \vdots \\
a_{N} & 0 & 0 & \ldots & p & b_{N} \\
c_{N} & 0 & 0 & \ldots & p & d_{N}
\end{array}\right)
$$

$$
\mathbb{B}=\left(\begin{array}{c}
R_{1} T_{1} \sum_{i \neq 1}^{d V} d m_{1 i} \\
-d Q_{1}+\sum_{i \neq 1}\left(h_{1 i}-h_{1}+R_{1} T_{1}\right) d m_{1 i} \\
\vdots \\
R_{N} T_{N} \sum_{i \neq N} d m_{N i} \\
-d Q_{N}+\sum_{i \neq N}\left(h_{N i}-h_{N}+R_{N} T_{N}\right) d m_{N i}
\end{array}\right)
$$

The state variable differentials, $d x$, are determined by solving the system of linear equations (9). The state 
variables can thereafter be calculated by numerical integration.

\section{SIMULATION ASPECTS}

There are some problems that have to be solved before equation system (9) can be used in a simulation. The problems are:

1. Non-existent zones - In the beginning of the combustion simulation, one or more zones may be non-existent. For example, there exist no burned gas zone before any gas has been combusted.

2. Ill-conditioned $\mathbb{A}$-matrix

3. Accuracy limitations due to finite precision arithmetic

4. Big relative error of $V_{i}$ and $m_{i}$, due to accumulation of calculation errors

Solutions to these problems for fixed gas composition are discussed below. The procedures can in most cases easily be extended to cover variable gas composition as well.

\subsection{Empty zones - Initializing a new zone}

Seeing that the determinant of $\mathbb{A}$ is

$$
\operatorname{det} \mathbb{A}=-p^{N-1} \prod_{i=1}^{N} m_{i} \cdot \sum_{j=1}^{N}\left(V_{j} c_{v, j} \prod_{k \neq j} c_{p, k}\right)
$$

there will always exist a unique solution to the system of linear equations as long as all $m_{i} \neq 0, i=1,2, \ldots, N$, since at least one $V_{i} \neq 0$. This means that once the combustion simulation has started, it runs smoothly using equation system (9). One question is what the temperature of the burned fuel is when $m_{b} \rightarrow 0^{+}$, which is the case at the start of combustion. Another problem when dealing with empty zones is to determine its state variable differentials, since the equation system can not be completely solved when $\mathbb{A}$ is singular.

3.1.1. Initial temperature of the burned gas The question about the initial temperature can be answered simply by studying the energy balance equation (2) and the ideal gas law (8) for zone $i$. At the initial state $m_{i}=V_{i}=0$ and $d Q_{i}=0$. It follows that:

$p d V_{i}+0 \cdot d p+0 \cdot d T_{i}=-0+\sum_{j \neq i}\left(h_{i j}-h_{i}+R_{i} T_{i}\right) d m_{i j}$

and

$$
p d V_{i}+0 \cdot d p+0 \cdot d T_{i}=R_{i} T_{i} \sum_{j \neq i} d m_{i j}
$$

Combining equation (11) and (12) gives:

$$
\begin{gathered}
R_{i} T_{i} \sum_{j \neq i} d m_{i j}=\sum_{j \neq i}\left(h_{i j}-h_{i}+R_{i} T_{i}\right) d m_{i j} \\
\mathbb{1} \\
\sum_{j \neq i}\left(h_{i j}-h_{i}\right) d m_{i j}=0
\end{gathered}
$$

To make this true for all mass flows, $h_{i j}$ has to be equal to $h_{i}$ as long as $d m_{i j} \neq 0$. Therefore, the initial temperature can be found by solving the equation $h_{i}\left(T_{i, i n i t}\right)=h_{i j}\left(T_{j}\right)$.

The equation $h_{i j}=h_{i}$ may not be solved analytically. If this is the case, the Newton-Raphson method can be useful:

$$
\left(T_{i}\right)_{n+1}=\left(T_{i}\right)_{n}-\frac{f\left(\left(T_{i}\right)_{n}\right)}{f^{\prime}\left(\left(T_{i}\right)_{n}\right)}
$$

where

$$
\begin{aligned}
& f\left(\left(T_{i}\right)_{n}\right)=h_{i}\left(\left(T_{i}\right)_{n}\right)-h_{i j}\left(T_{j}\right) \\
& f^{\prime}\left(\left(T_{i}\right)_{n}\right)=c_{p, i}\left(\left(T_{i}\right)_{n}\right)
\end{aligned}
$$

3.1.2. Solving the initial equation system If there is a zone without any mass, equation system (9) will not have any unique solution, and are thus to be modified. Combine the two rows of the equation system that describe the behavior of zone $i$, and eliminate $d T_{i}$. This gives

$$
\begin{gathered}
V_{i} d p+\gamma_{i} p d V=R_{i} T_{i} \sum_{j \neq i} d m_{i j}+m_{i} T_{i} d R_{i}+ \\
+\left(\gamma_{i}-1\right)\left(-d Q_{i}+\sum_{j \neq i}\left(h_{i j}-h_{i}+R_{i} T_{i}\right) d m_{i j}\right)
\end{gathered}
$$

where $\gamma_{i}=\frac{c_{v, i}+R_{i}}{c_{v, i}}$ (the ratio of specific heats). Set $m_{i}=0$, then the volume of zone $i$ is $V_{i}=0, h_{i j}=h_{i}$ and $d Q_{i}=0$. Equation (15) can thus be simplified into:

$$
p d V_{i}=\gamma_{i} R_{i} T_{i} \sum_{j \neq i} d m_{i j}
$$

The two rows for zone $i$ in the equation system are replaced with equation (16). Now, the resulting equation system consisting of $2 \mathrm{~N}$ equations can be solved.

The temperature of the zone in the next simulation step is given from the other state variables by use of the ideal gas law.

\subsection{Ill-conditioned matrix-Adaptive scaling}

There are large differences in magnitudes between the elements in the $\mathbb{A}$-matrix, since the pressure and temperature values are much greater than the volume and mass values. This makes scaling of the state variables necessary. By a fix scaling, the equation system will be solvable in most cases, but there is still a risk of getting a matrix that is so badly conditioned that the numerical solution to $\mathbb{A} x=\mathbb{B}$ will be highly unreliable. A better way to handle this is to use a scaling that adapts to the size of the state variables.

The temperatures and pressure change with about the same speed, while the volume and mass in each zone are highly correlated. An example of a feasible scaling is therefore: 


$$
\begin{array}{ll}
p^{\prime}=s_{p} p & d p^{\prime}=s_{p} d p \\
T^{\prime}=s_{p} T & d T^{\prime}=s_{p} d T \\
V_{i}^{\prime}=s_{i} V_{i} & d V_{i}^{\prime}=s_{i} d V_{i} \\
m_{i}^{\prime}=s_{i} m_{i} & d m_{i}^{\prime}=s_{i} d m_{i}
\end{array}
$$

where $s_{p}$ and $s_{i}$ are scaling factors determined in such a way that $p^{\prime}=1$ and $m_{i}^{\prime}=k_{i}, i=1,2, \ldots, N$ in each step. It can be shown that using this scaling has the same effect as multiplying the left hand side of each row, except the very first, in equation system (9), with a scaling factor $\left(s_{p} s_{i}\right)$. Thus, the rows on the right hand side have to be multiplied with the same scaling factors. The first row has to be treated somewhat differently. It is rewritten in the following way:

$$
\sum_{i=1}^{N} \frac{1}{s_{i}} d V_{i}^{\prime}=d V
$$

The scaled state variables $d x^{\prime}$ are determined by solving the resulting equation system $\mathbb{A}^{\prime} d x^{\prime}=\mathbb{B}^{\prime}$. Thereafter, $d x^{\prime}$ are transformed back to $d x$.

\subsection{Finite precision - Exclusion of zones}

If a zone is very small, it is difficult for the software in use to handle the calculations correctly. For example, the outcome of the calculation of $1-\epsilon$ is set to 1 even though $|\epsilon|>0$, if $\epsilon$ is a sufficiently small number. One of the consequences is that the mass of a zone might be set to zero even though it is still greater than zero.

A zone with no mass is the same as a non-existent zone, and according to (10) the $\mathbb{A}$-matrix is singular. Thus, the equations for the zone have to be excluded from the equation system. This is done by removing the two rows and two columns referring to the zone in the $\mathbb{A}$-matrix, the two rows in the $\mathbb{B}$-vector, and $d V_{i}$ and $d T_{i}$ from the list of differentiated state variables in $d x$.

\subsection{Accumulated faults - Consistency equations}

The model consists of a set of differential equations, which has to be solved numerically. There are several methods to do this, but regardless how well the chosen method performs, it will never be completely accurate. Tiny local errors in each step may result in a great relative global fault in the end, if the variable concerned decreases to a small value. A way of avoiding drifting of variables without reducing the time step, is to check the variables consistency and correct them if needed.

By using the ideal gas law, and knowing that the sum of all zone volumes is the same as the cylinder volume, the following equations are derived that can be used to check the consistency:

$$
\begin{aligned}
p & =\frac{m_{1} R_{1} T_{1}+\ldots+m_{N} R_{N} T_{N}}{V} \\
V_{1} & =\frac{m_{1} R_{1} T_{1}}{p} \\
& \vdots \\
V_{N} & =\frac{m_{N} R_{N} T_{N}}{p}
\end{aligned}
$$

\section{THE TWO-ZONE COMBUSTION MODEL}

To exemplify how the model can be used, it is applied on a two-zone system with fix gas compositions. In a two-zone system, the cylinder gas is divided into two zones; unburned $(u)$ and burned $(b)$ gas. The two zones are considered fully separate, and may therefore have different compositions as well as temperature. There is only one mass flow; a mass transport from the unburned to the burned zone. The gas is combusted at the moment when it leaves the unburned zone and enters the burned zone. This implies that $h_{u b}=h_{b u}=h_{u}$. Since changes in gas composition due to pressure and temperature are neglected, and the gas that enters the burned zone has the same composition as the gas already in the zone, $d R \equiv 0$.

According to the discussion above the multi-zone model can be simplified into the two-zone model (19).

\subsection{Simulation}

The two-zone model has been implemented and simulated in the crank angle domain. The mathematical tool used in the simulation was MATLAB 5.3. For the numerical solution to the differential equation, the MATLAB built-in function 'ode' was used. The following assumptions were made in addition to the previous ones:

1. The mass fraction burned is described by a Wiebe function (Heywood, 1988).

2. The Wiebe parameters $(m, a$ and $\Delta \theta$ ) are determined according to the method presented in (Eriksson, 1999), and the ignition angle is set to $20^{\circ}$.

3. The fuel becomes completely combusted during the combustion phase.

4. There is no heat transfer to the surroundings, nor between the zones.

5. The unburned zone contains a mixture of isooctane and air, which stochiometric air/fuel ratio is $\lambda=1$.

6. The burned gas consists of $14.0 \% \mathrm{H}_{2} 0,12.5 \%$ $\mathrm{CO}_{2}$, and $73.5 \% \mathrm{~N}_{2}$. Its gas constant is 292 .

7. The heat capacity and enthalpy are depending solely on temperature, not pressure (follows from the fixed gas composition assumption).

The equation system was scaled adaptively according to the method described in section 3.2, and then solved by Gaussian elimination. The volume of the unburned fuel $\left(V_{u}\right)$ risked getting a big relative error in the end 


$$
\begin{cases}d V_{u}+d V_{b} & =d V \\ V_{u} d p+p d V_{u}-m_{u} R_{u} d T_{u} & =R_{u} T_{u} d m_{u b} \\ p d V_{u}+m_{u} c_{v, u} d T_{u} & =-d Q_{u}+\left(h_{u b}-u_{u}\right) d m_{u b} \\ V_{b} d p+p d V_{b}-m_{b} R_{b} d T_{b} & =R_{b} T_{b} d m_{b u} \\ p d V_{b}+m_{b} c_{v, b} d T_{b} & =-d Q_{b}+\left(h_{b u}-u_{b}\right) d m_{b u}\end{cases}
$$

of the combustion phase. This was avoided thanks to the use of the consistency equation for $V_{u}$, as described in section 3.4. In the initial phase of the combustion the burned zone was non-existent as it had no mass. Therefore, the calculations had to be carried out as described in Section 3.1. The initial temperature of the burned gas, $T_{b}$, was determined by solving $h_{u}\left(T_{u}\right)=$ $h_{b}\left(T_{b}\right)$.

The simulation results are shown in Figure 1 and Figure 2. As can be seen, the shape of the simulated pressure curve and the measured pressure curve coincide quite well. The maximum pressures differ from each other, which could be expected since the heat transfer has been neglected in the simulation and fix gas composition is assumed. Another reason is that in a real engine, about $5 \%$ of the fuel remains unburned during the whole combustion. At $150^{\circ}$ after TDC, there is a pressure drop in the measured curve due to the gas exchange. This phase is not included in the simulation model, and therefore the pressure remains fairly constant at this angle. At about $50^{\circ}$ after TDC, the mass of the unburned zone is so small that numerical operations can not be carried out correctly (discussed in subsection 3.3). At this point, the two equations for the unburned zone are removed from equation system (19), leaving the equation system with only three equations - one of them being the trivial $d V_{b}=d V$.

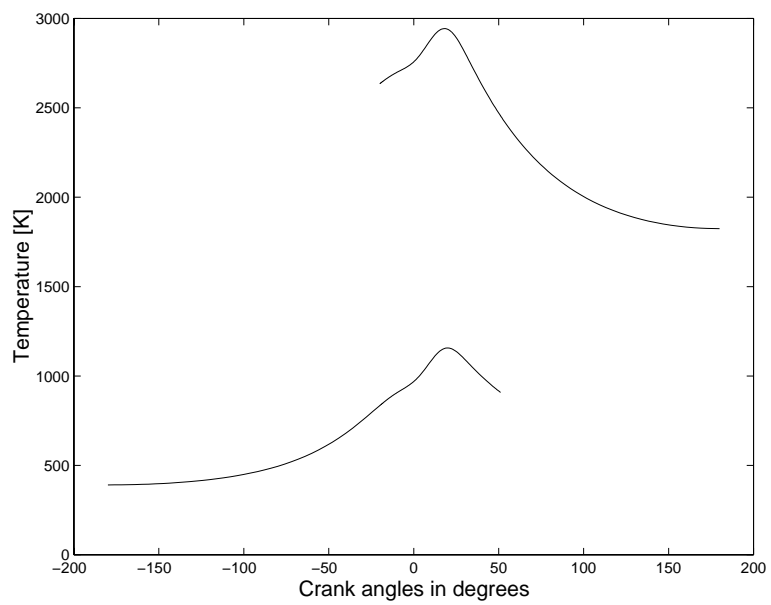

Fig. 1. Simulation results showing burned (upper curve) and unburned gas temperatures as function of crank angle. Inlet manifold pressure was set to $50 \mathrm{kPa}$, residual gas fraction to $7 \%$ and ignition angle to $20^{\circ}$ before TDC. The cylinder gas consists of a mixture of air and isooctan, with normalized air-fuel ratio $\lambda=1$. At about $50^{\circ}$ after TDC, the unburned zone is treated as non-existent.

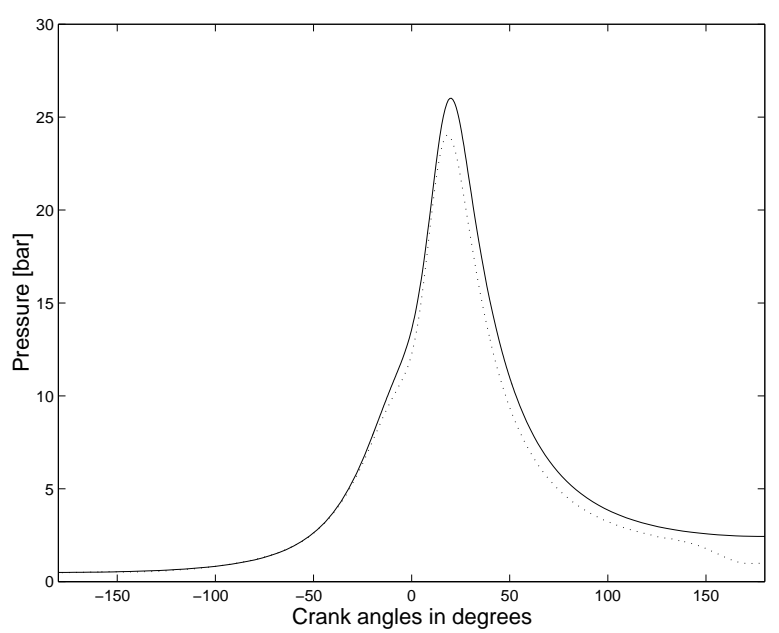

Fig. 2. Cylinder pressure as function of crank angle. The solid line shows the result from the simulation (same as in Figure 1), and the dashed line measured data from a SAAB 2.31 naturally aspirated engine with compression ratio $r_{c}=10.1$.

\subsection{The adaptive scaling}

The outcome of the simulation described in the previous subsection is used to show the effectiveness of the adaptive scaling. Without any kind of scaling, the condition number for the $\mathbb{A}$-matrix is $10^{16}$ or greater, which is shown in Figure 3. The condition number is very high in the beginning of the combustion simulation, caused by the small value of $m_{b}$. The local maximum at $20^{\circ}$ after TDC coincide with the pressure maximum. In the end of the combustion, the condition number increases rapidly again, since the mass of the unburned fuel $m_{u} \rightarrow 0$.

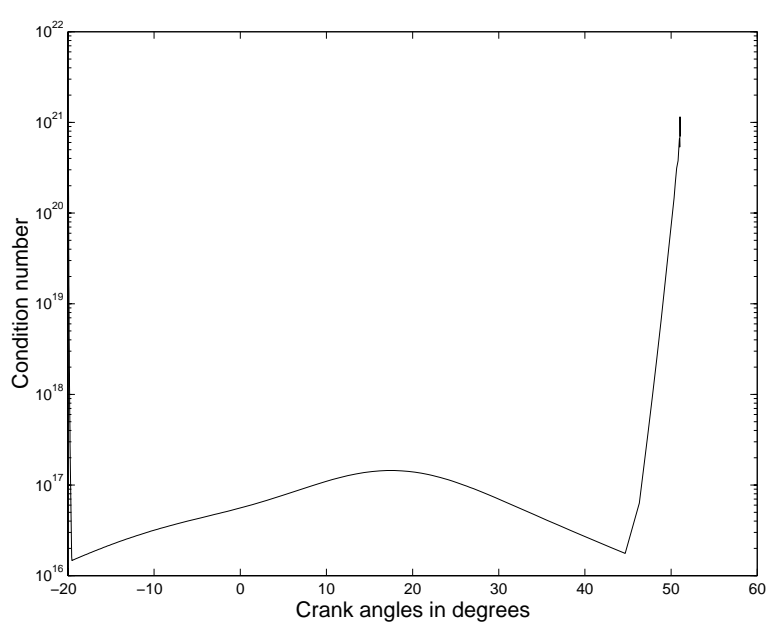

Fig. 3. Condition number for the unscaled $\mathbb{A}$-matrix.

In Figure 4, the $\mathbb{A}$-matrix has been scaled adaptively according to (17). Four different combinations of $k_{i^{-}}$ 
values are shown. If $k_{i}=R_{i}$, the $b_{i}$-values are equal to one, and is $k_{i}=c_{v, i}$ the $d_{i}$ values are equal to one. The lowest condition number is achieved in the latter case. For this choice of $k_{i}$ the condition number never gets as high as $5 \cdot 10^{4}$. Even with the worst choice of these four it stays beneath $5 \cdot 10^{7}$, which is a major improvement from the unscaled case.

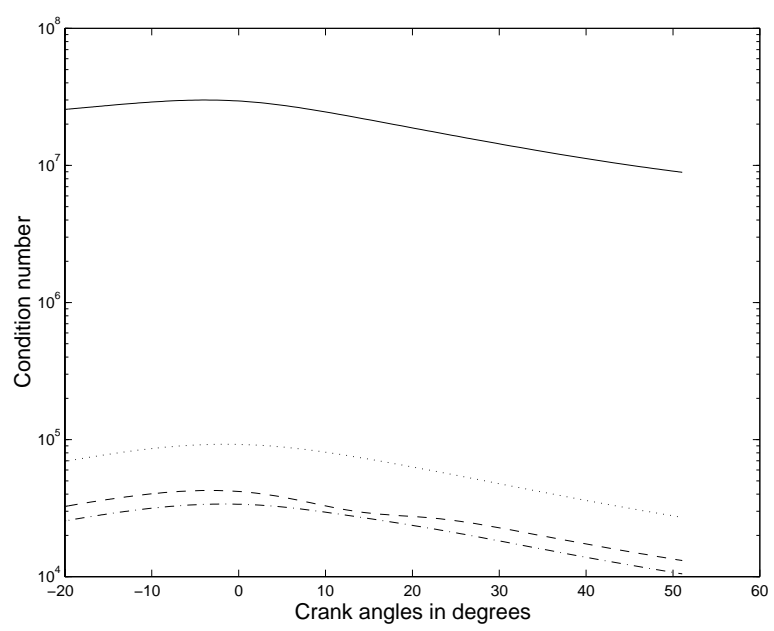

Fig. 4. Condition number for the $\mathbb{A}$-matrix after being scaled adaptively with $k_{u}=k_{b}=1$ (solid line), $k_{u}=R_{u}$ and $k_{b}=R_{b}$ (dotted), $k_{u}=c_{v, u}$ and $k_{b}=c_{v, b}$ (dash-dotted), and $k_{u}=k_{b}=3000$ (dashed).

\section{USE OF MODEL IN OTHER PHASES THAN THE COMBUSTION PHASE}

The multi-zone combustion model presented has a very flexible form. With no or minor changes it can be used to describe other phases than the combustion phase. For example, the compression and expansion phase can be simulated by applying the model to a one zone system where the burn speed $d x_{b} \equiv 0$. The compression and expansion phases in Figure 2 are simulated this way. Actually, what happens in section 4.1 when the twozone model is reduced into a one-zone model in the end of the simulation, is that the combustion phase turns into an expansion phase.

The gas exchange phase can be described with the multi-zone model as well. A way to do this is by divide the total system into a set of subsystems, that are represented by physical units like the cylinders and manifolds. During the gas exchange phase there is no combustion, but the gas composition will change due to that burned gas leaves the cylinders and are replaced by unburned gas.

\section{CONCLUSIONS}

A new formulation of zero-dimensional multi-zone models is presented. The formulation has a clear and simple structure which enables the user of the model to easily add and remove zones while maintaining the structure. A number of important issues that can give problems when simulating the model are also treated.

One issue is the initialization of a new zone. The most significant example of initialization is the initiation of the burned gas zone. It is shown how the state of the gas can be initiated in two steps, simply by studying the equations for the zone. Another issue arise due to the large differences in magnitude between the variables. This can result in numerical problems when simulating the system on a computer with finite precision. It is shown that the numerical difficulties can be avoided by proper scaling of the variables. The final issue is how faults, accumulated during the simulation, can be reduced by utilizing a set of consistency equations. For each zone one consistency equation can be formulated using the ideal gas law.

Simulation results from a two-zone model are compared with measured engine pressure data, which illustrates how the presented formulation can be applied. The simulation result is used to indicate the strength of the adaptive scaling.

\section{REFERENCES}

Eriksson, Lars (1999). Spark advance for optimal efficiency. SAE Technical Paper no.99-01-0548.

Eriksson, Lars (2001). Documentation for the chemical equilibrium program package. Forthcoming, february.

Finn, C.B.P. (1998). Thermal Physics. second edition ed.. Stanley Thornes (publisherss) Ltd.

Heywood, J. B. (1988). Internal Combustion Engine Fundamentals. McGraw-Hill series in mechanical engineering. McGraw-Hill.

Keating, Eugene L. (1993). Applied Combustion. number ISBN 0-8247-8127-9. Marcel Dekker, INC.

Kittel, Charles and Herbert Kroemer (1980). Thermal Physics. second edition ed.. W. H. Freeman and Company.

Ramos, J. I. (1989). Internal Combustion Engine Modeling. number ISBN 0-89116-157-0. Hemisphere Publishing Corporation.

Sonntag, Richard E., Claus Borgnakke and Gordon J. Van Wylen (1998). Fundamentals of Thermodynamics. 5 ed.. John Wiley \& Sons.

Turns, Stephen R. (2000). An Introduction to Combustion - Concepts and Applications. Mechanical Engineering Series. second ed.. McGraw Hill. 